\title{
An unusual iron Lo-BAL quasar detected by ISOCAM*
}

\author{
P.-A. Duc ${ }^{1,2}$, P. B. Hall ${ }^{3}$, D. Fadda ${ }^{4}$, P. Chanial ${ }^{2}$, D. Elbaz ${ }^{2}$, P. Monaco ${ }^{5}$, E. Pompei $^{6}$, B. M. Poggianti ${ }^{7}$, \\ H. Flores ${ }^{8}$, A. Franceschini ${ }^{9}$, A. Biviano ${ }^{10}$, A. Moorwood ${ }^{11}$, and C. Cesarsky ${ }^{11}$
}

1 CNRS URA 2052

2 CEA, DSM, DAPNIA, Service d'Astrophysique, 91191 Gif-sur-Yvette Cedex, France

3 Pontificia Universidad Católica de Chile, Departamento de Astronomía y Astrofísica, Casilla 306, Santiago 22, Chile and Princeton University Observatory, Princeton, NJ 08544-1001, USA

4 Instituto de Astrofisica de Canarias, Via Lactea s/n, 38200 La Laguna - Tenerife, Spain

5 Dipartimento di Astronomia, via G.B. Tiepolo 11, 34131 Trieste, Italy

6 European Southern Observatory, Santiago

7 Osservatorio Astronomico di Padova, vicolo dell'Osservatorio 5, 35122 Padova, Italy

8 DAEC/LUL, Observatoire de Paris-Meudon, 5 place Jules Janssen, 92195 Meudon, France

9 Dipartimento di Astronomia, Università di Padova, Vicolo dell'Osservatorio, 5, 35122 Padova, Italy

10 INAF - Osservatorio Astronomico di Trieste, via G.B. Tiepolo, 11, 34131 Trieste, Italy

11 European Southern Observatory, Karl-Schwarzschild-Strasse, 2, 85748 Garching bei München, Germany

Received 8 March 2002 / Accepted 2 May 2002

\begin{abstract}
We report the discovery of an unusual low-ionization broad absorption line quasar at $z=1.776$ which exhibits absorption lines from many excited states of Fe II. This member of the rare class of "FeLoBAL" quasars was serendipitously found in a mid-infrared (MIR) survey of distant clusters carried out with the ISOCAM camera. ISO J005645.1-273816 has a high MIR to UV luminosity ratio, suggesting a strong dust obscuration plus emission from very hot dust. This characteristic makes MIR surveys particularly efficient at detecting LoBAL quasars.
\end{abstract}

Key words. quasars: absorption lines - quasars: individual: ISO J005645.1-273816 - infrared: galaxies

\section{Introduction}

About $10 \%$ of optically selected quasars show absorption from gas with blueshifted outflow velocities of typically $\lesssim 0.1 c$ (Weymann et al. 1991). These Broad Absorption Line (BAL) quasars may just be normal quasars seen along a particular line of sight, such that most quasars have BAL outflows covering $\sim 10-30 \%$ of the sky, with mass loss rates comparable to the quasar accretion rates $\left(\sim 1 M_{\odot} \mathrm{yr}^{-1}\right)$. It is also possible that $\mathrm{BAL}$ quasars represent a dust-enshrouded early phase in the lives of most, if not all, quasars (Becker et al. 2000). In either case, BAL outflows must be understood to understand quasars as a whole.

BAL quasars are divided into three observational subtypes depending on what type of absorption is seen. HiBAL quasars show absorption from high-ionization

Send offprint requests to: P.-A. Duc,

e-mail: paduc@cea.fr

* Based on observations with ISO, an ESA project with instruments funded by ESA Member States with the participation of ISAS and NASA and observations collected at the European Southern Observatory, Paranal, Chile (ESO No 65.O-0563 A), at La Silla, Chile (ESO No 67.A-0253 A). lines like C IV. LoBAL quasars (Voit et al. 1993) also show absorption from low-ionization lines like $\mathrm{Mg}$ II. FeLoBAL quasars (Becker et al. 1997) are LoBAL quasars which also show absorption from excited Fe II or Fe III. Populations of unusual BAL quasars with extremely strong or complex absorption have recently been found through followup of FIRST radio sources (Becker et al. 1997, 2000; Menou et al. 2001; Lacy et al. 2002), z> 4 quasar candidates from the Digitized Palomar Sky Survey (Djorgovski et al. 2001), and color-selected quasar candidates from the Sloan Digital Sky Survey (Hall et al. 2002). These unusual BAL quasars suggest that the range of physical conditions present in BAL outflows is larger than previously suspected.

In this paper we report the discovery of another such unusual BAL quasar with the Infrared Space Observatory (ISO). What little data exists on the midto far-IR properties of BAL quasars suggests that BAL quasars, and especially LoBAL quasars, may be much more common or have larger covering fractions than suggested by optical surveys. For example, the dusty LoBAL quasar Hawaii 167 was discovered in a near-IR survey covering only 77 square arcmin (Cowie et al. 1994), and $\sim 20_{-10}^{+15 \%}$ of all IRAS-selected quasars are LoBAL 
quasars (Weymann et al. 1991; Low et al. 1989; Boroson \& Meyers 1992) vs. only $\sim 1.5 \%$ in optical surveys. Dusty gas with a nearly $100 \%$ covering factor in LoBAL quasars helps explain many of their unusual properties, including ubiquitous signs of recent mergers in their host galaxies (Canalizo \& Stockton 2001).

\section{Observations}

The quasar ISO J005645.1-273816 (hereafter ISO 0056-2738) was serendipitously discovered as part of an on-going study of distant clusters of galaxies mapped in the mid-infrared by the ISOCAM camera on board the ISO satellite. This quasar was found in the field of the $z=0.56$ cluster J1888.16CL.

J1888.16CL was observed with ISOCAM at 6.75 and $15 \mu \mathrm{m}$ in December 1997 and with ISOPHOT at $200 \mu \mathrm{m}$ in December 1996. The ISOCAM data were processed following the method presented by Fadda et al. (2000) which uses extensive simulations based of the addition of fake sources to the science images to estimate flux reconstruction factors as well as error estimates. ISOPHOT data were reduced with the standard pipeline PIA (Gabriel et al. 1997).

$B$ and $R$ images of J1888.16CL were obtained in August and September 1999 with the WFI camera installed on the MPG/ESO 2.2m telescope at La Silla, as part of a program on supernova monitoring (PI: Patat). Near-infrared $J, H$ and $K$ s broad-band images of the field were taken in August 2001 with SOFI on the NTT at La Silla. We retrieved a F814W image from the HST archives. The optical spectrum of ISO 0056-2738 was obtained in September 2000 with the FORS1 instrument installed on the VLT UT1/Antu. The medium-resolution grism $300 \mathrm{~V}$ (resolution 420) covering the wavelength range $4200 \AA-$ $8820 \AA$ was used associated with a slitlet of $1.2^{\prime \prime}$ positioned with the MOS unit.

\section{Results}

\subsection{Source identification}

With a flux of $1.3 \mathrm{mJy}$, ISO 0056-2738 is one of the brightest $15 \mu \mathrm{m}$ extragalactic sources in the field of J1888.16CL. It is unambiguously associated with an object which appears unresolved in the optical and near-infrared bands. On the HST image, its FWHM is lower than $0.22^{\prime \prime}$, and no clear extension is visible (see Fig. 1).

The basic properties of the object, including position and photometry, are listed in Table 1. ISO 0056-2738 was not detected by IRAS at 60 and $100 \mu \mathrm{m}$, nor at $200 \mu \mathrm{m}$ by ISOPHOT or at $20 \mathrm{~cm}$ by the NRAO VLA Sky Survey (NVSS).

\subsection{Spectroscopic analysis}

The VLT spectrum of ISO 0056-2738 is shown in Fig. 2. Extensive UV absorption plus narrow emission from FeII UV1 and MgII identifies it as an unusual

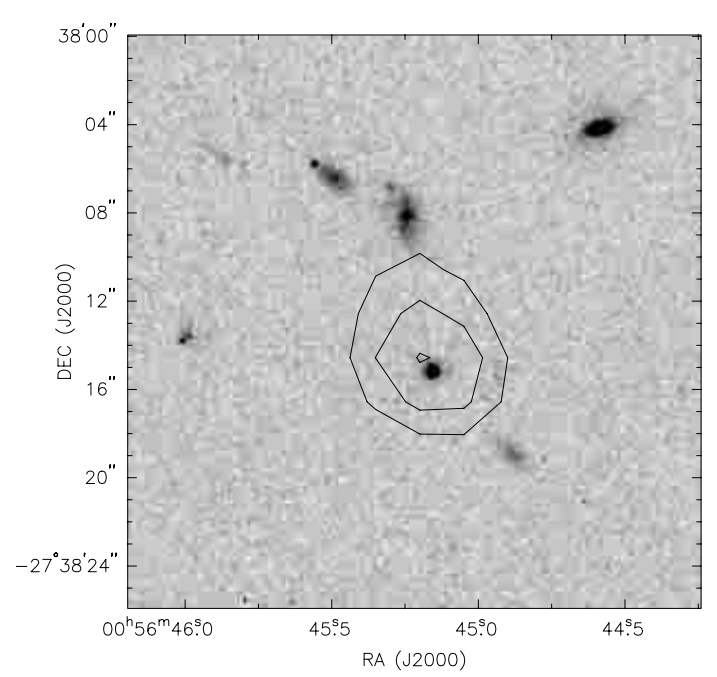

Fig. 1. ISOCAM LW3 $(15 \mu \mathrm{m})$ contours superimposed on a combined F606W+F814W HST image centered on the quasar. The levels are 2, 2.5 and $3 \mu \mathrm{Jy} \operatorname{arcsec}^{-2}$.

Table 1. Data.

\begin{tabular}{ll}
\hline \hline RA $(\mathrm{J} 2000)$ & $00: 56: 45.15$ \\
DEC $(\mathrm{J} 2000)$ & $-27: 38: 15.6$ \\
\hline$B(0.46 \mu \mathrm{m})$ & $22.74 \pm 0.03 \mathrm{mag}$ \\
$R(0.65 \mu \mathrm{m})$ & $20.95 \pm 0.02 \mathrm{mag}$ \\
$J(1.25 \mu \mathrm{m})$ & $18.29 \pm 0.04 \mathrm{mag}$ \\
$H(1.65 \mu \mathrm{m})$ & $17.68 \pm 0.04 \mathrm{mag}$ \\
$K \mathrm{~s}(2.16 \mu \mathrm{m})$ & $17.16 \pm 0.04 \mathrm{mag}$ \\
LW2 $(6.75 \mu \mathrm{m})$ & $0.51 \pm 0.15 \mathrm{mJy}$ \\
LW3 $(15 \mu \mathrm{m})$ & $1.33 \pm 0.33 \mathrm{mJy}$ \\
PHOT $(200 \mu \mathrm{m})$ & $<1 \mathrm{Jy}$ \\
VLA NVSS $(20 \mathrm{~cm})$ & $<2.3 \mathrm{mJy}$ \\
\hline
\end{tabular}

FeLoBAL quasar similar to FIRST 0840+3633 (Becker et al. 1997) and especially PSS $1537+1227$ (Djorgovski et al. 2001).

We adopt a systemic redshift of $z=1.776 \pm 0.002$ based on the narrow emission lines of C IV, Fe II 2627 (multiplet UV1) and Mg II, using the effective wavelengths for those transitions in the first SDSS composite quasar (Vanden Berk et al. 2001). As long as the relative velocity shifts of the lines in this quasar do not differ greatly from those quasars used to construct the composite, this should yield consistent redshifts for all lines. Indeed, the three redshifts agree to better than $1 \sigma$. Of course, since all three emission lines are affected by absorption, the true systematic emission line redshift may lie blueward of our adopted value. The balnicity index (BI; Weymann et al. 1991) of this object, measured from $\mathrm{Al}$ III, is a small but nonzero $305 \mathrm{~km} \mathrm{~s}^{-1}$. The less restrictive absorption index (AI; Hall et al. 2002) is $2860 \mathrm{~km} \mathrm{~s}^{-1}$. Both measures assume the $\mathrm{Al}$ III trough is at least $6280 \mathrm{~km} \mathrm{~s}^{-1}$ wide, though it could be confused with a different trough beyond $4000 \mathrm{~km} \mathrm{~s}^{-1}$.

At least two different redshift systems are present in the absorption. The C IV and MgII troughs appear to reach zero flux at $1.7487 \pm 0.0008$. 


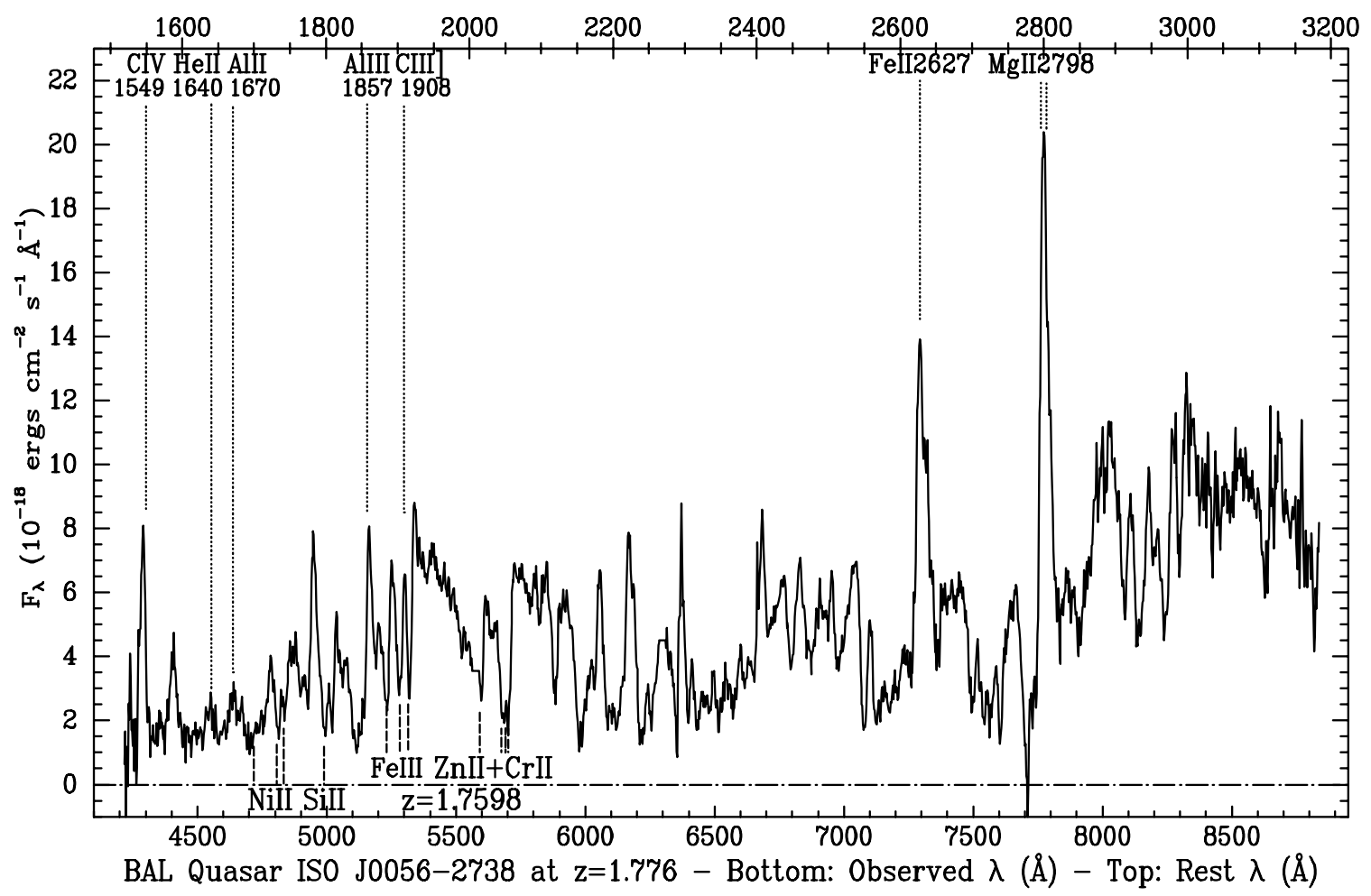

Fig. 2. The VLT spectrum of ISO 0056-2738. The horizontal dot-dashed line is the zero flux level. Dotted lines above the spectrum show the wavelengths of the strong emission lines labeled across the top of the plot (including the expected wavelength of the C III] line peak). Dashed lines below the spectrum show the wavelengths of the strong absorption lines from the $z=1.7598$ system labeled across the bottom of the plot.

The narrow absorption from the Fe III UV34 multiplet $(\lambda \lambda \lambda 1895.46,1914.06,1926.30)$ gives $z=1.7598 \pm 0.0009$. There are no obvious features in the $\mathrm{CIV}$ or $\mathrm{Mg}$ II troughs at this redshift, but there is absorption from Ni II $\lambda \lambda \lambda 1709,1741,1751, \quad$ Si II $\lambda 1808$, and Zn II $\lambda \lambda 2026,2062$ plus Cr II $\lambda \lambda \lambda 2056,2062,2066$.

Overall, however, the spectrum is dominated by absorption from ground and excited terms of FeII. Essentially every absorption trough not labelled in Fig. 2 can be identified with Fe II. For example, the strong absorption just redward of C IV at $1550-1600 \AA$ is from Fe II UV multiplets 44-46 (Wampler et al. 1995; Hall et al. 2002). Fe II multiplets from excited atomic terms are present up to at least UV191 ( $\sim 1787 \AA$, excitation potential $\sim 2.88 \mathrm{eV}$ ). There may also be excited Cr II UV5-UV8 absorption near 2675 and $2835 \AA$ (de Kool et al. 2002). The $3130 \AA$ trough could have contributions from even more highly excited Cr II and possibly O III $\lambda 3133$, as discussed in Sect. 5.1.3 of Hall et al. (2002).

Given the strength of the Fe II absorption, the Mg II absorption must be saturated. However, the flux in the absorption troughs does not reach zero except for narrow regions in the C IV and Mg II troughs. This means that the absorbing region does not cover the continuum source except at a narrow range of velocities, and that the Fe II absorbing region probably has a smaller partial covering factor than the Mg II and C IV regions.

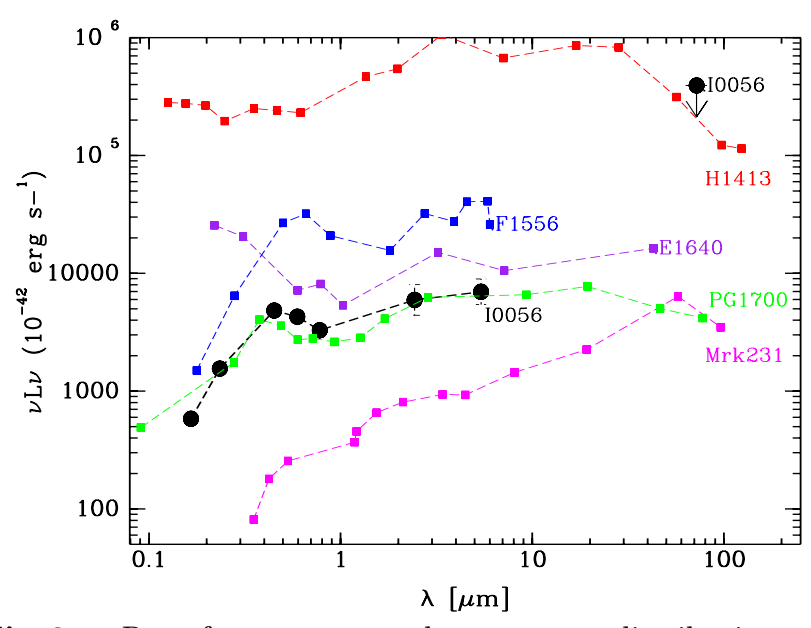

Fig. 3. Rest-frame spectral energy distribution of ISO J005645.1-273816 (plain circles) and, for comparison, of other BAL quasars with IR data: Mrk 231 (LoBAL; $z=0.04$; data from NED); PG 1700+518 (LoBAL; $z=0.292$; Andreani et al. 1999), ELAIS J164010+410502 (BAL; $z=1.099$; Morel et al. 2001); FIRST J155633.7+351757 (LoBAL; $z=1.48$; Clavel 1998), H1413+117 (BAL; $z=2.54$; Barvainis et al. 1995). Note the lines joining the data points, indicated by plain squares, were drawn to guide the eye and by no means represent the real SEDs. We used $H_{0}=75 \mathrm{~km} \mathrm{~s}^{-1} \mathrm{Mpc}^{-1}$ and $q_{0}=0.5$.

\section{Discussion and conclusions}

The spectral energy distribution of ISO 0056-2738 in the rest-frame UV to infrared domain is compared in Fig. 3 
to that of a sample of BAL quasars for which mid and far infrared fluxes are available in the literature.

The SED of ISO 0056-2738 appears to be similar, in shape and luminosity, to that of the nearby optically selected low ionization BAL quasar PG $1700+518$, at least within the wavelength range for which data are available for both objects. If this similarity extends to the far infrared where IRAS fluxes are available for PG $1700+518$, one would infer for ISO 0056-2738 a total infrared luminosity of $L_{\mathrm{IR}}=4 \times 10^{12} L_{\odot}$. Instead one may use as template Mrk 231, which is known as the nearest galaxy with a LoBAL signature, and has a strong IR bump at $60 \mu \mathrm{m}$. Taking the rest-frame $5 \mu \mathrm{m}$ luminosity as the normalizing factor, the total infrared luminosity of ISO 0056-2738 becomes $2 \times 10^{13} L_{\odot}$. The PHOT measurement at $200 \mu \mathrm{m}$ flux indicates that this is most likely an upper limit. One may conclude for this analysis that unless its SED plunges at $\lambda>10 \mu \mathrm{m}$, ISO 0056-2738 belongs to the class of ULIRGs and may even be a hyperluminous infrared galaxy, like many quasars (e.g. Haas et al. 2000). ISO 0056-2738 lies behind a rich cluster of galaxies; however the distance from the cluster center, about $3.5^{\prime}$, is such that a gravitational lensing by the cluster is unlikely.

The spectral energy distribution of the quasar in the rest-frame ultraviolet is much redder than that of the SDSS composite quasar of Vanden Berk et al. (2001). Using the SMC extinction curve of Prevot et al. (1984), we estimate a large but not unprecedented extinction of $E(B-V)=0.25 \pm 0.05$. Besides, ISO 0056-2738 seems to share with the few other LoBAL quasars with available mid-IR data (see Fig. 3) a steep UV to MIR spectral index. In addition to the extinction in the UV, its very high MIR to UV luminosity ratio (rest-frame $\nu L_{\nu}(5.40 \mu \mathrm{m}) /$ $\left.\nu L_{\nu}(0.16 \mu \mathrm{m})=12\right)$ is likely due to the presence of unusually large quantities of very hot dust at $1000 \mathrm{~K}$, the emission of which peaks at about $3 \mu \mathrm{m}$. Becker et al. (1997) claim that a relative excess of radio emission could be another property of LoBAL quasars. Note that ISO $0056-2738$ is not likely to be radio-loud $\left(\log \left(R^{*}\right)>\right.$ 1). It has $\log \left(R^{*}\right)<1.79$ using the Becker et al. (2000) definition with $\alpha_{\text {rad }}=-0.5$, but corrected for reddening of $E(B-V)=0.25$ it has intrinsic $\log \left(R^{*}\right)<1.05$.

To our knowledge, ISO 0056-2738 is the first FeLoBAL quasar so far directly found at mid-infrared wavelengths ${ }^{1}$. At optical wavelengths, the SDSS Early Data Release Quasar Sample (Schneider et al. 2002) only contains four FeLoBAL quasars out of 200-300 BAL quasars at $1.485<z<3.9$ where both HiBAL and LoBAL quasars can be selected via SDSS spectra, and three or four more FeLoBAL quasars at lower redshift (Hall et al. 2002). Therefore the probability of a serendipitous discovery with ISOCAM of such FeLoBAL quasars is then extremely low unless mid-IR surveys are very efficient in detecting such objects. A quasar like ISO 0056-2738 would

\footnotetext{
1 However several BAL quasars were identified in the course of the $15 \mu \mathrm{m}$ European Large Area ISO Survey (ELAIS) (e.g. Morel et al. 2001; Alexander et al. 2001).
}

likely have been missed by most optical surveys since it does not show any prominent broad emission lines and has no UV excess. The infrared surveys with SIRTF such as GOODS and SWIRE should soon confirm whether the number of FeLoBAL quasars has been underestimated in optically-based surveys.

Acknowledgements. PBH acknowledges financial support from Chilean grant FONDECYT/1010981. We are grateful to our referee, M. Lacy, for his comments which helped clarifying the paper. We thank G. Rodighiero for her help in reducing the PHOT data, E. Cappellaro and F. Patat for providing us the optical images of J1888.16CL.

\section{References}

Alexander, D. M., La Franca, F., Fiore, F., et al. 2001, ApJ, 554,18

Andreani, P., Franceschini, A., \& Granato, G. 1999, MNRAS, 306, 161

Barvainis, R., Antonucci, R., Hurt, T., Coleman, P., \& Reuter, H.-P. 1995, ApJ, 451, L9

Becker, R. H., Gregg, M. D., Hook, I. M., et al. 1997, ApJ, 479, L93

Becker, R. H., White, R. L., Gregg, M. D., et al. 2000, ApJ, 538, 72

Boroson, T. A., \& Meyers, K. A. 1992, ApJ, 397, 442

Canalizo, G., \& Stockton, A. 2001, ApJ, 555, 719

Clavel, J. 1998, A\&A, 331, 853

Cowie, L. L., Songaila, A., Hu, E. M., et al. 1994, ApJ, 432, L83

de Kool, M., Becker, R. H., Gregg, M. D., White, R. L., \& Arav, N. 2002, ApJ, 567, 58

Djorgovski, S. G., Brunner, R. J., Mahabal, A. A., et al. 2001, in Mining the Sky, ed. A. J. Banday, S. Zaroubi, \& M. Bartelmann (Berlin: Springer-Verlag), in press [astro-ph/0012489]

Fadda, D., Elbaz, D., Duc, P.-A., et al. 2000, A\&A, 361, 827

Gabriel, C., Acosta-Pulido, J., Heinrichsen, I., Morris, H., \& Tai, W.-M. 1997, in ASP Conf. Ser. 125, Astronomical Data Analysis Software and Systems VI, 6, 108

Haas, M., Müller, S. A. H., Chini, R., et al. 2000, A\&A, 354, 453

Hall, P. B., Anderson, S. F., Strauss, M. A., et al. 2002, ApJS, in press [astro-ph/0203252]

Lacy, M., Gregg, M., Becker, R., et al. 2002, AJ, in press [astro-ph/0203065]

Low, F. J., Cutri, R. M., Kleinmann, S. G., \& Huchra, J. P. 1989, ApJ, 340, L1

Menou, K., Vanden Berk, D. E., Ivezić, Ž., et al. 2001, ApJ, 561,645

Morel, T., Efstathiou, A., Serjeant, S., et al. 2001, MNRAS, 327,1187

Prevot, M. L., Lequeux, J., Prevot, L., Maurice, E., \& Rocca-Volmerange, B. 1984, A\&A, 132, 389

Schneider, D. P., Richards, G. T., Fan, X., et al. 2002, AJ, 123, 567

Vanden Berk, D. E., Richards, G. T., Bauer, A., et al. 2001, AJ, 122, 549

Voit, G. M., Weymann, R. J., \& Korista, K. T. 1993, ApJ, 413, 95

Wampler, E. J., Chugai, N. N., \& Petitjean, P. 1995, ApJ, 443, 586

Weymann, R. J., Morris, S. L., Foltz, C. B., \& Hewett, P. C. 1991, ApJ, 373, 23 